VOL. 57 (1998) [73-91]

\title{
COMPACT QUANTUM GROUPS AND THEIR COREPRESENTATIONS
}

\author{
HuU Hung BuI
}

\begin{abstract}
A compact quantum group is defined to be a unital Hopf $C^{*}$-algebra generated by the matrix elements of a family of invertible corepresentations. We present a version of the Tannaka-Krein duality theorem for compact quantum groups in the context of abstract categories; this result encompasses the result of Woronowicz and the classical Tannaka-Krein duality theorem. We construct the orthogonality relations (similar to the case of compact groups). The Plancherel theorem is then established.
\end{abstract}

\section{INTRODUCTION}

The Gelfand-Naimark theorem provides a contravariant equivalence $X \longmapsto C(X)$ between the category of compact Hausdorff spaces and the category of commutative unital $C^{*}$-algebras. It is known that every compact Lie group is isomorphic as a topological group to a compact subgroup of $G L(n, \mathbf{C})$ and that every compact group is a projective limit of compact Lie groups. Woronowicz defined in [14] a compact matrix pseudogroup to be a unital Hopf $C^{*}$-algebra generated by the matrix elements of one invertible matrix; and hence it can be viewed as a compact quantum Lie group. Following [3] and [11], we define a compact quantum group as the inductive limit of a family of Woronowicz's compact matrix pseudogroups.

Monoidal categories now form the right framework for the study of quantum groups, see $[6,7,8,9,16]$. In this paper, we generalise the duality theorem of Woronowicz in $[15]$ to the case of our compact quantum groups. This is a noncommutative version of the classical Tannaka-Krein duality theorem. Here we deal with abstract categories in contrast to these two last results, which were treated in the context of the category of finite dimensional Hilbert spaces; see also [4]. Another proof, which has more categorical flavour and is based on the result of Joyal and Street [6], is given in [2].

Woronowicz proved in [14, Section 5] that the matrix elements of two nonequivalent irreducible corepresentations are orthogonal, but the orthogonality of the matrix elements of each irreducible corepresentation was left open there. In this paper we show

Received 14th April, 1997

This research was supported by an ARC Small Grant at Macquarie University. The author would like to thank Professor R. Street for his support.

Copyright Clearance Centre, Inc. Serial-fee code: 0004-9729/98 \$A2.00+0.00. 
that with a suitable basis, the matrix elements of each irreducible corepresentation are orthogonal. Our formula here is completely similar to the case of compact groups. We define the Fourier transformation and establish the Plancherel theorem using these orthogonality relations.

Our work is organised as follows. In Section 1 we define a compact quantum group to be a unital Hopf $C^{*}$-algebra generated by the matrix elements of a family of invertible corepresentations. In Section 2 we establish the existence of the Haar measure on compact quantum groups. With the Haar measure it is quite easy to decompose an invertible corepresentation into irreducible subcorepresentations. Then we gather some important properties of unitary corepresentations, which say that the category of finite dimensional unitary corepresentations has subobjects and direct sums. The role of the coinverse is completely ignored in this section. In Section 3 we prove a version of the Tannaka-Krein duality theorem for our compact quantum groups. We follow [6] by replacing concrete monoidal $C^{*}$-categories in $[15]$ by abstract monoidal $C^{*}$-categories equipped with faithful monoidal linear *-functors. In Section 4 we use the material established in Section 3 to prove the orthogonality relations for our compact quantum groups. We show that the Fourier transformation is a unitary.

\section{Compact quantum groups}

Let $A_{0}$ be a ${ }^{*}$-algebra with a unit $I$. A unital *-homomorphism $\Delta: A_{0} \longrightarrow A_{0} \otimes A_{0}$ is called a comultiplication on $A_{0}$ if $(\Delta \otimes i d) \circ \Delta=(i d \otimes \Delta) \circ \Delta$. Let $V$ be a finite dimensional vector space and let $B(V)$ denote the algebra of linear maps on $V$. An element $\alpha \in B(V) \otimes A_{0}$ is called a corepresentation of $A_{0}$ on $V$ if

$$
(i d \otimes \Delta)(\alpha)=(\alpha \otimes I)(i d \otimes \tau)(\alpha \otimes I)
$$

Here $\tau$ denotes the twist map $\tau(a \otimes b)=b \otimes a$. Pick a basis $\left\{e_{i}\right\}$ for $V$ and let $\left\{e_{i}^{*}\right\}$ denote the dual basis for the vector space $V^{*}$ of linear functionals on $V$. We define linear maps $\theta_{i, j}: V \longrightarrow V$ by $\theta_{i, j}(v)=e_{j}^{*}(v) e_{i}$. Then for any $\alpha \in B(V) \otimes A_{0}$, there is a unique matrix $\{\alpha(\widehat{\imath, j})\} \in M_{n}\left(A_{0}\right)$ such that $\alpha=\sum_{i, j} \theta_{i, j} \otimes \alpha(i, j)$. We refer to $\alpha(i, j),(i, j=1, \ldots, n)$, as the matrix elements of $\alpha$ with respect to the basis $\left\{e_{i}\right\}$. We say that $(\alpha, V)$ is a unitary corepresentation if $V$ is a Hilbert space and $\alpha$ is a unitary of the *-algebra $B(V) \otimes A_{0}$.

Let $\left(\alpha_{1}, V_{1}\right)$ and $\left(\alpha_{2}, V_{2}\right)$ be corepresentations of $A_{0}$. A linear map $T \in B\left(V_{1}, V_{2}\right)$ is said to intertwine $\alpha_{1}$ and $\alpha_{2}$ if $(T \otimes I) \alpha_{1}=\alpha_{2}(T \otimes I)$. The set of all linear maps intertwining $\alpha_{1}$ and $\alpha_{2}$ is denoted by $\operatorname{Hom}\left(\alpha_{1}, \alpha_{2}\right)$. We say that $\alpha_{1}$ and $\alpha_{2}$ are equivalent if $\operatorname{Hom}\left(\alpha_{1}, \alpha_{2}\right)$ contains an invertible element. The monoidal product of $\alpha_{1}$ and $\alpha_{2}$ is defined by 


$$
\alpha_{1} \diamond \alpha_{2}=(i d \otimes \tau)\left(\alpha_{1} \otimes I\right)\left(I \otimes \alpha_{2}\right) .
$$

Let $P_{i} \in B\left(V_{1} \oplus V_{2}, V_{i}\right)$ and $E_{i} \in B\left(V_{i}, V_{1} \oplus V_{2}\right)$ be the canonical projections and embeddings. The direct sum of $\alpha_{1}$ and $\alpha_{2}$ is defined by

$$
\alpha_{1} \oplus \alpha_{2}=\sum_{i=1}^{2}\left(E_{i} \otimes I_{B}\right) \alpha_{i}\left(P_{i} \otimes I_{B}\right)
$$

Note that $P_{i} \in \operatorname{Hom}\left(\alpha_{1} \oplus \alpha_{2}, \alpha_{i}\right)$ and $E_{i} \in \operatorname{Hom}\left(\alpha_{i}, \alpha_{1} \oplus \alpha_{2}\right)$.

Let $W$ be a subspace of $V$ and let $P \in B(V, W)$ and $E \in B(W, V)$ be the canonical projection and embedding. Set $\beta=(P \otimes I) \alpha(E \otimes I) \in B(W) \otimes A_{0}$. Then $(E \otimes I) \beta=\alpha(E \otimes I)$ if and only if $\alpha(v \otimes I) \in W \otimes A_{0}$ for all $v \in W$. In this case, $(\beta, W)$ is a corepresentation of $A_{0}$; we call $W$ an $\alpha$-invariant subspace and call $(\beta, W)$ a subcorepresentation of $(\alpha, V)$.

Let $(\alpha, V)$ be a corepresentation of $A_{0}$. By a conjugate space of $V$, we mean a vector space $\widetilde{V}$ together with a bijective antilinear map $J: V \longrightarrow \widetilde{V}$. For each $T \in B(V)$, we define $\widetilde{T} \in B(\widetilde{V})$ by $\widetilde{T}(J v)=J(T v)$. The conjugate $(\widetilde{\alpha}, \widetilde{V})$ of $(\alpha, V)$ is defined by $\tilde{\alpha}=\alpha^{J \otimes *}$.

Let $F$ be a family of finite dimensional corepresentations of $A_{0}$. We denote by $\mathcal{R}_{F}$ the smallest class of corepresentations that contains $F$ and that is closed under monoidal products, direct sums, subcorepresentations, conjugates and equivalent corepresentations. Then each element $\alpha$ in $\mathcal{R}_{F}$ can be written in the form

$$
\alpha=\sum_{i=1}^{n}\left(R_{i} \otimes I\right) \alpha_{i}\left(S_{i} \otimes I\right)
$$

where each $\alpha_{i}$ is the monoidal product of a finite family of elements of $F$ or $\{\widetilde{\beta}: \beta \in$ $F\}, S_{i} \in \operatorname{Hom}\left(\alpha, \alpha_{i}\right)$ and $R_{i} \in \operatorname{Hom}\left(\alpha_{i}, \alpha\right)$ such that $\sum_{i=1}^{n} R_{i} S_{i}=I_{\alpha}$.

PROPOSITION 1.1. Suppose that $F$ is a family of finite dimensional corepresentations of $A_{0}$. Let $A_{F}$ denote the unital *-subalgebra generated by the matrix elements of all elements of $F$. Then $A_{F}$ is the linear span of elements $(f \otimes i d)(\alpha)$, where $f \in B\left(V_{\alpha}\right)^{*}$ and $\alpha$ is the monoidal product of a finite family of elements of $F$ or $\{\widetilde{\beta}: \beta \in F\}$.

The proof of this proposition is similar to that of [14, Proposition 2.5].

The following definition is an analogue of the $C^{*}$-algebra of continuous functions on a compact group. See [3, Définition 4.1].

DEFINITION 1.2: Let $A$ be a unital $C^{*}$-algebra equipped with a comultiplication $\Delta$. We say that $A=(A, \Delta)$ is a compact quantum semigroup if there is a family $F$ of 
finite dimensional corepresentations of $A$ such that the unital *-subalgebra $A_{F}$ generated by the matrix elements of all elements of $F$ is dense in $A$. A compact quantum semigroup $A$ is called a compact quantum group if there is an antimultiplicative linear map $\kappa: A_{F} \longrightarrow A_{F}$ such that $\kappa\left(\kappa\left(a^{*}\right)^{*}\right)=a$ for all $a \in A_{F}$, and $(i d \otimes \kappa)(\alpha)=\alpha^{-1}$ for all $\alpha \in F$.

We shall refer to $(A, \Delta, F, \kappa)$ as a compact quantum group. We call $\kappa$ the coinverse of $A$.

REMARK 1.3. (a) By arguments similar to those in [14, Proposition 1.8] we can show that there is a unique ${ }^{*}$-homomorphism $\varepsilon: A_{F} \longrightarrow \mathbf{C}$ such that

$$
\mu \circ(\kappa \otimes i d) \circ \Delta=\mu \circ(i d \otimes \kappa) \circ \Delta=\varepsilon,
$$

where $\mu: A \otimes A \longrightarrow A$ is defined by $\mu(a \otimes b)=a b$. Furthermore we have

$$
\mu \circ(\varepsilon \otimes i d) \circ \Delta=\mu \circ(i d \otimes \varepsilon) \circ \Delta=i d .
$$

Therefore $\left(A_{F}, \Delta, \varepsilon, \kappa\right)$ is a Hopf *-algebra in the sense of [12, Definition 2.1].

(b) By arguments similar to those in $[\mathbf{1 4}$, Proposition 1.9] we can show that

$$
\Delta \circ \kappa=\tau \circ(\kappa \otimes \kappa) \circ \Delta .
$$

\section{The HAar measure AND UNITARY COREPRESENTATIONS}

Throughout this section, $A=(A, \Delta)$ is a unital $C^{*}$-algebra equipped with a comultiplication $\Delta$; and we shall refer to it as a Hopf $C^{*}$-algebra. The space of all continuous linear functionals of $A$ is denoted by $A^{*}$. The convolution of $\phi, \psi \in A^{*}$ is defined by

$$
\phi * \psi=(\phi \otimes \psi) \circ \Delta
$$

We denote by $\phi^{* n}$ the $n^{\text {th }}$ convolution power $\phi * \cdots * \phi$.

The following result contains the result $[\mathbf{1 4}$, Proposition 2.7] as a special case. Here we use the Markov-Kakutani fixed point theorem instead of the Cesàro limit.

Proposition 2.1. Suppose that $A=(A, \Delta)$ is a unital Hopf $C^{*}$-algebra. Then for any state $\phi$ of $A$, there exists a state $\lambda_{\phi}$ of $A$ such that

$$
\phi * \lambda_{\phi}=\lambda_{\phi}=\lambda_{\phi} * \phi .
$$

Proof: Recall that the space $\mathcal{S}$ of all states of $A$ is a convex compact set with respect to the weak *-topology. Fix a $\phi \in \mathcal{S}$. We define $L, R: \mathcal{S} \longrightarrow \mathcal{S}$ by $L(\eta)=\phi * \eta$ and $R(\eta)=\eta * \phi$. Then $L$ and $R$ are continuous affine maps and $L R=R L$. Therefore by the Markov-Kakutani fixed point theorem [10, Theorem 5.23], there exists $\lambda_{\phi} \in \mathcal{S}$ such that $L\left(\lambda_{\phi}\right)=\lambda_{\phi}=R\left(\lambda_{\phi}\right)$, and this proves the desired result.

Using Proposition 1.1 and Proposition 2.1, the proof of the following result is similar to that of $[14$, Proposition 4.1]. 
Proposition 2.2. Suppose that $A=(A, \Delta, F)$ is a compact quantum semigroup. Assume that for any $\alpha$ in $F, \alpha$ and $\widetilde{\alpha}$ are invertible. Let $\phi$ be a faithful state of $A$. Then there exists a unique state $\lambda_{\phi}$ of $A$ such that

$$
\eta * \lambda_{\phi}=\eta(I) \lambda_{\phi}=\lambda_{\phi} * \eta, \quad \forall \eta \in A^{*}
$$

The following result generalises the result [14, Theorem 4.2].

THEOREM 2.3. Suppose that $A=(A, \Delta, F)$ is a compact quantum semigroup. Assume that for any $\alpha$ in $F, \alpha$ and $\widetilde{\alpha}$ are invertible. Then there exists a unique state $\lambda$ of $A$ such that

$$
\eta * \lambda=\eta(I) \lambda=\lambda * \eta, \quad \forall \eta \in A^{*}
$$

Proof: Let $M$ be a finite subset of $F$ and let $A(M)$ denote the unital $C^{*}$ subalgebra generated by the matrix elements of all $\alpha \in M$. Since $A(M)$ is separable, $A(M)$ admits a faithful state. Thus by Proposition 2.2 there exists a unique state $\lambda_{M}$ of $A(M)$ such that for any $\xi \in A(M)^{*}$, we have

$$
\lambda_{M} * \xi=\xi * \lambda_{M}=\xi(I) \lambda_{M}
$$

Now observe that $A_{F}$ is the union of all $A_{M}$ where $M$ are finite subsets of $F$. For any finite subsets $M$ and $N$ of $F$, by the uniqueness of $\lambda_{M}$ and $\lambda_{N}$, they are just the restrictions of $\lambda_{M \cup N}$ to $A(M)$ and $A(N)$. Thus for any $a \in A_{M} \cap A_{N}$, we have $\lambda_{M}(a)=\lambda_{N}(a)$. Therefore we can define a linear functional $\lambda_{0}$ on $A_{F}$ by $\lambda_{0}(a)=\lambda_{M}(a)$ if $a \in M$. Clearly $\lambda_{0}$ is bounded on $A_{F}$ and hence extends to a bounded linear functional $\lambda$ on $A$. Let $\eta \in A^{*}$. For any $a \in A_{M}$, we have

$$
\begin{aligned}
(\eta * \lambda)(a) & =(\eta \otimes \lambda)(\Delta(a))=\left(\eta_{M} \otimes \lambda_{M}\right)(\Delta(a)) \\
& =\left(\eta_{M} * \lambda_{M}\right)(a)=\eta_{M}(I) \lambda_{M}(a)=\eta(I) \lambda(a)
\end{aligned}
$$

Therefore $\eta * \lambda=\eta(I) \lambda$. The other equality can be proved in the same way, and hence we get the desired result.

We shall refer to the state $\lambda$ as the Haar measure for the compact quantum semigroup $(A, \Delta, F)$.

We remark that the existence of the Haar measure can be proven in a more general context, see [13].

By using the Haar measure $\lambda$, we can show that for any invertible finite dimensional corepresentation $(\alpha, V)$ of $A$, there exists a structure of Hilbert space on $V$ so that $\alpha$ becomes a unitary corepresentation on $V$; see [14, Theorem 5.2]. 
THEOREM 2.4. Any finite dimensional unitary corepresentation of a unital Hopf $C^{*}$-algebra $A=(A, \Delta)$ is the direct sum of irreducible unitary subcorepresentations.

PROOF: Let $(\alpha, V)$ be a finite dimensional unitary corepresentation of $A$. Since $\operatorname{Hom}(\alpha, \alpha)$ is a finite dimensional $C^{*}$-algebra, there are minimal projections $\mu_{i} \in$ $\operatorname{Hom}(\alpha, \alpha)$ such that $\mu_{i} \mu_{j}=\delta_{i, j}$ and $\sum_{i=1}^{n} \mu_{i}=I_{\alpha}$. Put $V_{i}=\mu_{i}(V)$ and let $P_{i} \in$ $B\left(V, V_{i}\right)$ be the canonical projections. Since $\mu_{i} \in \operatorname{Hom}(\alpha, \alpha)$, it follows that

$$
\left(\mu_{i} \otimes I\right) \alpha\left(P_{i}^{*} \otimes I\right)=\alpha\left(P_{i}^{*} \otimes I\right) .
$$

Therefore $V_{i}$ are $\alpha$-invariant subspaces, and hence $\alpha_{i}=\left(P_{i} \otimes I\right) \alpha\left(P_{i}^{*} \otimes I\right)$ are subcorepresentations of $A$ on $V_{i}$. Since $\alpha$ is unitary and $P_{i} P_{i}^{*}=I_{V_{i}}$ and $P_{i}^{*} P_{i}=$ $\mu_{i} \in \operatorname{Hom}(\alpha, \alpha)$, it follows that $\alpha_{i}$ are unitaries. Since $\mu_{i} \mu_{j}=\delta_{i, j}$, it follows that $P_{i} P_{j}^{*}=\delta_{i, j}$ and then $\alpha$ is the direct sum of $\alpha_{i}$. Since $\mu_{i}$ are minimal, we deduce that $\alpha_{i}$ are irreducible.

By using the coinverse $\kappa$, the result [14, Theorem 4.5] is now immediately from Theorem 2.4.

Theorem 2.5. Let $A=(A, \Delta, F)$ be as in Theorem 2.3. Suppose that $\mathcal{L}$ is a family of finite dimensional irreducible unitary corepresentations of $A$ such that the matrix elements of all elements of $\mathcal{L}$ generate a dense subspace $A_{\mathcal{L}}$ of $A$. Then every finite dimensional irreducible unitary corepresentation of $A$ is equivalent to an element of $\mathcal{L}$.

Proof: Let $\left(\beta, V_{\beta}\right)$ be any finite dimensional unitary corepresentation of $A$. We claim that $\operatorname{Hom}(\alpha, \beta) \neq\{0\}$ for some $\alpha$ in $\mathcal{L}$. Suppose that $\operatorname{Hom}(\alpha, \beta)=\{0\}$ for all $\alpha$ in $\mathcal{L}$. Pick an orthonormal basis $\left\{e_{i}^{\beta}\right\}$ for $V_{\beta}$, and define $\theta_{i, j}^{\beta}(u)=\left\langle u \mid e_{j}^{\beta}\right\rangle e_{i}^{\beta}$ for $u \in V_{\beta} ;$ and similarly for each $\left(\alpha, V_{\alpha}\right)$ in $\mathcal{L}$. We write

$$
\beta=\sum_{i, j} \theta_{i, j}^{\beta} \otimes \beta(i, j), \quad \alpha=\sum_{i, j} \theta_{i, j}^{\alpha} \otimes \alpha(i, j) .
$$

Put $\gamma=\beta \diamond \widetilde{\alpha}$ and $E=(i d \otimes \lambda)(\gamma)$. Then

$$
\begin{aligned}
& \gamma=\sum_{i, j, k, l} \theta_{k, l}^{\beta} \otimes \widetilde{\theta_{i, j}^{\alpha}} \otimes \beta(k, l) \alpha(i, j)^{*} \\
& E=\sum_{i, j, k, l} \lambda\left(\beta(k, l) \alpha(i, j)^{*}\right) \theta_{k, l}^{\beta} \otimes \widetilde{\theta_{i, j}^{\alpha}} .
\end{aligned}
$$

Identify $B\left(V_{\alpha}, V_{\beta}\right)$ with $V_{\beta} \otimes \widetilde{V}_{\alpha}$ by the bijective linear map $T \longmapsto \sum_{i} T e_{i}^{\alpha} \otimes \widetilde{e_{i}^{\alpha}}$, and we then show that

$$
\gamma(T \otimes I)=\beta(T \otimes I) \alpha^{*}, \quad \forall T \in B\left(V_{\alpha}, V_{\beta}\right)
$$


Let $\xi \in V_{\beta} \otimes \tilde{V}_{\alpha}$ and let $\phi \in A^{*}$. Since $\phi * \lambda=\phi(I) \lambda$, it follows that

$$
\phi(I) E \xi=(i d \otimes \phi)(\gamma) E \xi=(i d \otimes \phi)\left(\beta(E \xi \otimes I) \alpha^{*}\right) .
$$

Therefore $E \xi \in \operatorname{Hom}(\alpha, \beta)=\{0\}$. It then follows that $E=0$, and hence

$$
\lambda\left(\beta(k, l) \alpha(i, j)^{*}\right)=0, \quad \forall i, j, k, l .
$$

Since $A_{\mathcal{L}}$ is dense in $A$, we get

$$
\lambda\left(\beta(k, l) a^{*}\right)=0, \quad \forall k, l, \quad \forall a \in A .
$$

Therefore $(i d \otimes \lambda)\left(\beta \beta^{*}\right)=0$. On the other hand, $(i d \otimes \lambda)\left(\beta \beta^{*}\right)=I_{\beta}$ since $\beta$ is a unitary. This contradiction proves the claim.

Now suppose that $\left(\beta, V_{\beta}\right)$ is irreducible. Pick a nonzero $T \in \operatorname{Hom}(\alpha, \beta)$ for some $\alpha$ in $\mathcal{L}$. Since $\alpha$ is irreducible, $T^{*} T=c I_{\alpha}$ for some $c>0$. We then deduce that $c^{-1 / 2} T$ is a unitary of $\operatorname{Hom}(\alpha, \beta)$.

REMARK 2.6. (a) Let $A=(A, \Delta, F)$ be as in Theorem 2.3. Pick a complete set $\mathcal{J}$ of mutually nonequivalent irreducible corepresentations belonging to $\mathcal{R}_{F}$. For each $\left(\alpha, V_{\alpha}\right)$ in $\mathcal{J}$, let $\alpha(i, j),(i, j=1, \ldots, \operatorname{dim}(\alpha))$, denote the matrix elements of $\alpha$ with respect to a basis of $V_{\alpha}$. Using Proposition 1.1 and Theorem 2.4, we can show that $A_{F}$ is the vector space generated by the set

$$
\{\alpha(i, j): i, j=1, \ldots, \operatorname{dim}(\alpha), \alpha \in \mathcal{J}\} .
$$

By arguments similar to those in [14, Lemma 4.8], we deduce that this set is a basis for $A_{F}$.

(b) Let $A=(A, \Delta, F)$ be a compact quantum semigroup. Assume that every element of $F$ is unitary. Then $A_{F}$ is the linear span of elements $(f \otimes i d)(\alpha)$, where $f \in B\left(V_{\alpha}\right)^{*}$ and $\alpha$ is the monoidal product of a finite family of elements of $F$ or $\left\{\beta^{*}: \beta \in F\right\}$. We then deduce that Proposition 2.2 and Theorem 2.3 still hold.

\section{TANNAKA-KREIN DUALITY FOR COMPACT QUANTUM GROUPS}

Throughout this section, $\mathcal{R}$ is a strict monoidal $C^{*}$-category with subobjects and direct sums in the sense of $[4$, Section 1$]$. We denote by $\mathcal{H i l}_{f}$ the strict monoidal $C^{*}$ category of finite dimensional Hilbert spaces; here the monoidal product is the usual tensor product, and the monoidal unit is the complex numbers C. If $Q=(Q, \Lambda, \mathcal{L}, \jmath)$ is a compact quantum group, we denote by $\mathcal{U} \operatorname{corep}_{f}(Q)$ the strict monoidal $C^{*}$-category of finite dimensional unitary corepresentations of $Q$. 
We recall from [7, Definition 7.2] that a monoidal category $\mathcal{C}$ is said to be left autonomous if for any object $r$, there is an object $\widetilde{r}$ and there are arrows $\vartheta_{r} \in \operatorname{Hom}(\iota, r \tilde{r})$ and $\bar{\vartheta}_{r} \in \operatorname{Hom}(\widetilde{r} r, \iota)$ such that

$$
\begin{aligned}
& \left(I_{r} \diamond \bar{\vartheta}_{r}\right)\left(\vartheta_{r} \diamond I_{r}\right)=I_{r}, \\
& \left(\bar{\vartheta}_{r} \diamond I_{\tilde{r}}\right)\left(I_{\tilde{r}} \diamond \vartheta_{r}\right)=I_{\tilde{r}} ;
\end{aligned}
$$

where $\diamond$ is the monoidal product, and $\iota$ is the monoidal unit of $\mathcal{C}$. We call $\tilde{r}$ a left dual of $r$, and we refer to the pair $\left(\vartheta_{r}, \bar{\vartheta}_{r}\right)$ as an adjunction between $\widetilde{r}$ and $r$. If $\mathcal{C}$ is a $C^{*}$-category then $r$ is a left dual of $\tilde{r}$ with an adjunction $\left(\bar{\vartheta}_{r}^{*}, \vartheta_{r}^{*}\right)$. Note that $\mathcal{C}$ is also right autonomous.

The category $\mathcal{H}_{i l_{f}}$ is left autonomous in the following way. For each object $V$ of

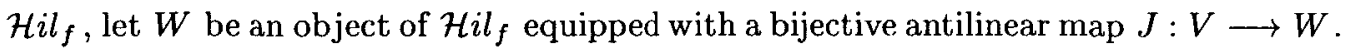
Pick an orthonormal basis $\left\{e_{i}\right\}$ for $V$, and define

$$
\begin{aligned}
t_{J}(1) & =\sum e_{i} \otimes J e_{i}, \\
\bar{t}_{J}\left(J v_{2} \otimes v_{1}\right) & =\left\langle v_{1} \mid v_{2}\right\rangle, \quad \forall v_{1}, v_{2} \in V .
\end{aligned}
$$

Then $W$ is a left dual of $V$ with an adjunction $\left(t_{J}, \bar{t}_{J}\right)$. Also $V$ is a left dual of $W$ with an adjunction $\left(\bar{t}_{J}^{*}, t_{J}^{*}\right)$. Note that $\bar{t}_{J}^{*}=t_{J-1}$ and $t_{J}^{*}=\bar{t}_{J-1}$, with respect to an orthonormal basis $\left\{f_{i}\right\}$ of $W$.

If $Q$ is a compact quantum group, then the category $\mathcal{U}_{\operatorname{corep}}(Q)$ is left autonomous in the following way. For each object $\left(\alpha, V_{\alpha}\right)$ of $\mathcal{U}_{\operatorname{corep}}(Q)$, put $\tilde{\alpha}=\alpha^{J \otimes *}$, where $J$ is a bijective antilinear map from $V_{\alpha}$ onto a vector space $W$. Define an inner product on $W$ so that $(\widetilde{\alpha}, W)$ is a unitary corepresentation. Since $\alpha$ is unitary, it follows that $t_{J} \in \operatorname{Hom}(\iota, \alpha \diamond \widetilde{\alpha})$ and $\bar{t}_{J} \in \operatorname{Hom}(\widetilde{\alpha} \diamond \alpha, \iota)$. Thus $(\widetilde{\alpha}, W)$ is a left dual of $\left(\alpha, V_{\alpha}\right)$ with an adjunction $\left(t_{J}, \bar{t}_{J}\right)$.

The following result is a generalisation of the classical Tannaka-Krein duality theorem and the result of Woronowicz [15, Theorem 1.3]. See also [6, Section 7, Theorem $3]$ and [2, Theorem 2.5].

THEOREM 3.1. Suppose that $\mathcal{R}$ is a left autonomous strict monoidal $C^{*}$ category with subobjects and direct sums. Assume that there is a faithful monoidal linear *-functor $H: \mathcal{R} \longrightarrow \mathcal{H i l}_{f}$. Then $\mathcal{R}$ is equivalent to $\mathcal{U}$ corep $_{f}(Q)$ for some compact quantum group $Q=(Q, \Lambda, \mathcal{L}, \jmath)$.

The proof of this theorem will follow from Theorem 3.3 and 3.4.

Our next purpose is to establish the notation for Theorem 3.3. The context here is more general and more elegant than that in [15]. Our effort is to characterise 
$\mathcal{U}_{\text {corep }}(Q)$ as an abstract category similar to [4]. The proof of Theorem 3.3 is very similar to proofs in [15], and for convenience we reproduce some main arguments here.

Let $r$ be an object of $\mathcal{R}$. Since $H: \mathcal{R} \longrightarrow \mathcal{H}_{i l_{f}}$ is faithful, $\operatorname{Hom}(r, r)$ is a finite dimensional $C^{*}$-algebra. Hence there are minimal projections $\mu_{i} \in \operatorname{Hom}(r, r)$ such that $\mu_{i} \mu_{j}=\delta_{i j}$ and $\sum_{i} \mu_{i}=I_{r}$. Since $\mathcal{R}$ has subobjects, there are arrows $\nu_{i} \in \operatorname{Hom}\left(r_{i}, r\right)$ such that and $\nu_{i}^{*} \nu_{i}=I_{r_{i}}$ and $\nu_{i} \nu_{i}^{*}=\mu_{i}$. Thus $r=\sum_{i}^{\oplus} r_{i}$. Since $\left\{\mu_{i}\right\}$ are minimal, $\left\{r_{i}\right\}$ are irreducible. Therefore every object of $\mathcal{R}$ can be decomposed into the direct sum of a finite number of irreducible objects.

Put $t_{r}=H\left(\vartheta_{r}\right)$ and $\bar{t}_{r}=H\left(\bar{\vartheta}_{r}\right)$. Since $H$ is monoidal, $\left(t_{r}, \bar{t}_{r}\right)$ is an adjunction between $H(\widetilde{r})$ and $H(r)$. Let $\widetilde{J}: H(r) \longrightarrow \widetilde{H(r)}$ be the canonical antilinear map. Then $\left(\bar{t}_{r} \otimes I\right)\left(I \otimes t_{\tilde{J}}\right)$ is a bijective linear map from $H(\tilde{r})$ onto $\widetilde{H(r)}$, and hence $\operatorname{dim}(H(\widetilde{r}))=\operatorname{dim}(\widehat{H(r)})$. Therefore there is a bijective antilinear map $J: H(r) \longrightarrow$ $H(\widetilde{r})$ such that $\left\langle J u_{1} \mid J u_{2}\right\rangle=\left\langle u_{2} \mid u_{1}\right\rangle$ for all $u_{1}, u_{2} \in H(r)$. Put $\psi=\left(\bar{t}_{r} \otimes I\right)\left(I \otimes t_{J}\right)$, then $\psi$ is a bijective linear map on $H(\widetilde{r})$. We then deduce that

$$
t_{r}=\left(I \otimes \psi^{-1}\right) t_{J}, \quad \bar{t}_{r}=\bar{t}_{J}(\psi \otimes I) .
$$

Put $J_{r}=\psi^{-1} J$. Then we have

$$
t_{r}=t_{J_{r}}, \quad \bar{t}_{r}=\bar{t}_{J_{r}} .
$$

Put $t_{\tilde{r}}=\bar{t}_{r}^{*}$ and $\bar{t}_{\tilde{r}}=t_{r}^{*}$. Since $H$ is a ${ }_{\text {-functor, we get }}$

$$
t_{\tilde{r}}=H\left(\vartheta_{\tilde{r}}\right), \quad \bar{t}_{\tilde{r}}=H\left(\bar{\vartheta}_{\tilde{r}}\right) .
$$

We define a model for $(\mathcal{R}, H)$ to be a pair $\left(Q_{0}, \Gamma\right)$ consisting of a unital *-algebra $Q_{0}$ and a family of unitaries $\Gamma_{r} \in B(H(r)) \otimes Q_{0}$ such that

$$
\begin{aligned}
\Gamma_{r s} & =\Gamma_{r} \circ \Gamma_{s}, \\
(H(\mu) \otimes I) \Gamma_{r} & =\Gamma_{s}(H(\mu) \otimes I),
\end{aligned}
$$

for all objects $r, s$ and $\mu \in \operatorname{Hom}(r, s)$. A model $\left(Q_{0}, \Gamma\right)$ is called a universal model if $Q_{0}$ is generated by the matrix elements of all $\Gamma_{r}$, and for any model $\left(B_{0}, \Pi\right)$ there is a homomorphism $\Phi: Q_{0} \longrightarrow B_{0}$ of unital *-algebras such that $(i d \otimes \Phi)\left(\Gamma_{r}\right)=\Pi_{r}$ for all $r$. If $\left(Q_{0}, \Gamma\right)$ and $\left(B_{0}, \Pi\right)$ are universal models then there is an isomorphism $\Phi: A_{0} \longrightarrow B_{0}$ of unital *-algebras such that $(i d \otimes \Phi)\left(\Gamma_{r}\right)=\Pi_{r}$ for all $r$.

Let $\mathcal{J}(\mathcal{R})$ be a complete set of mutually nonequivalent irreducible objects; we assume that $\iota \in \mathcal{J}(\mathcal{R})$. Set

$$
Q_{0}=\sum^{\oplus}\left\{B(H(r))^{*}: r \in \mathcal{J}(\mathcal{R})\right\} .
$$


For each $r \in \mathcal{J}(\mathcal{R})$, the canonical embedding $f_{r} \longmapsto\left[f_{r}\right]$ from $B(H(r))^{*}$ into $Q_{0}$ is linear, and hence there exists a unique $\Gamma_{r} \in B(H(r)) \otimes Q_{0}$ such that $\left[f_{r}\right]=\left(f_{r} \otimes i d\right)\left(\Gamma_{r}\right)$. Note that if $\left\{E_{i, j}^{r}\right\}$ is a basis for $B(H(r))$, and $\left\{\omega_{i, j}^{r}\right\}$ is the dual basis for $B(H(r))^{*}$, that is $\omega_{i, j}^{r}\left(E_{k, l}^{r}\right)=\delta_{i k} \delta_{j l}$, then we have

$$
\Gamma_{r}=\sum_{i, j} E_{i, j}^{r} \otimes\left[\omega_{i, j}^{r}\right]
$$

and $\left\{\left[\omega_{i, j}^{r}\right]: i, j=1, \ldots, \operatorname{dim}(H(r)), r \in \mathcal{J}(\mathcal{R})\right\}$ is a basis for $Q_{0}$.

For each $r \in \mathcal{J}(\mathcal{R})$, we pick an orthonormal basis $\left\{e_{i}^{r}\right\}$ for $H(r)$. We define

$$
\theta_{i, j}^{r}(u)=\left\langle u \mid e_{j}^{r}\right\rangle e_{i}^{r}, \quad \forall u \in H(r) .
$$

Then $\left\{\theta_{i, j}^{r}\right\}$ is a basis for $B(H(r))$. We denote by $\left\{\varpi_{i, j}^{r}\right\}$ the dual basis of $\left\{\theta_{i, j}^{r}\right\}$ for $B(H(r))^{*}$. We define

$$
e_{i, j}^{r}(u)=\left\langle u \mid e_{j}^{r}\right\rangle J_{r}^{-1}\left(e_{i}^{\tilde{r}}\right), \quad \forall u \in H(r)
$$

Then $\left\{e_{i, j}^{r}\right\}$ is a basis for $B(H(r))$. We denote by $\left\{\varepsilon_{i, j}^{r}\right\}$ the dual basis of $\left\{e_{i, j}^{r}\right\}$ for $B(H(r))^{*}$. If we write

$$
e_{j}^{r}=\sum_{i} \chi^{r}(i, j) J_{r}^{-1}\left(e_{i}^{\tilde{r}}\right)
$$

then we get

$$
\begin{aligned}
\theta_{i, j}^{r} & =\sum_{k} \chi^{r}(k, i) e_{k, j}^{r}, \\
\varepsilon_{i, j}^{r} & =\sum_{k} \chi^{r}(k, i) \varpi_{k, j}^{r} .
\end{aligned}
$$

Let $r$ be any object of $\mathcal{R}$. Pick a decomposition $\sum_{i=1}^{n} \mu_{i} \mu_{i}^{*}=I_{r}$ with $\mu_{i} \epsilon$ $\operatorname{Hom}\left(r_{i}, r\right)$ and $r_{i} \in \mathcal{J}(\mathcal{R})$. We define

$$
\Gamma_{r}=\sum_{i=1}^{n}\left(H\left(\mu_{i}\right) \otimes I\right) \Gamma_{r_{i}}\left(H\left(\mu_{i}^{*}\right) \otimes I\right) .
$$

Then for any objects $r, s$ and any $\mu \in \operatorname{Hom}(r, s)$, we have

$$
(H(\mu) \otimes I) \Gamma_{r}=\Gamma_{s}(H(\mu) \otimes I) .
$$


We define a multiplication operation on $Q_{0}$ by

$$
\left[f_{r}\right]\left[f_{s}\right]=\left(f_{r} \otimes f_{s} \otimes i d\right)\left(\Gamma_{r s}\right),
$$

for all $f_{r} \in B(H(r))^{*}, f_{s} \in B(H(s))^{*}$ and $r, s \in \mathcal{J}(\mathcal{R})$. Then $Q_{0}$ becomes an algebra with a unit $I=\left[\varepsilon^{\iota}\right]$. For any objects $r$ and $s$, we have

$$
\Gamma_{r s}=\Gamma_{r} \diamond \Gamma_{s}
$$

Let $r \in \mathcal{J}(\mathcal{R})$. For any $T \in B(H(r))$ and for any $f_{r} \in B(H(r))^{*}$, we define $\widetilde{T} \in B(H(\widetilde{r}))$ and $\tilde{f}_{r} \in B(H(\widetilde{r}))^{*}$ by

$$
\tilde{T}\left(J_{r} u\right)=J_{r}(T u), \quad \tilde{f}_{r}(\tilde{T})=\overline{f(T)} .
$$

We define an involution operation on $Q_{0}$ by

$$
\left[f_{r}\right]^{*}=\left[\tilde{f}_{r}\right],
$$

for all $f_{r} \in B(H(r))^{*}$ and $r \in \mathcal{J}(\mathcal{R})$. Then $Q_{0}$ becomes a unital *-algebra. Each $\Gamma_{r}$ is a unitary of $B(H(r)) \otimes Q_{0}$.

Let $\left(B_{0}, \Pi\right)$ be any model for $(\mathcal{R}, H)$. Put

$$
\Phi\left(\left[f_{r}\right]\right)=\left(f_{r} \otimes i d\right)\left(\Pi_{r}\right), \quad \forall f_{r} \in B(H(r))^{*} .
$$

Then $\Phi$ is a homomorphism of unital *-algebras, and $(i d \otimes \Phi)\left(\Gamma_{r}\right)=\Pi_{r}$ for all $r$. Thus $\left(Q_{0}, \Gamma\right)$ is a universal model for $(\mathcal{R}, H)$. If we put

$$
\Theta_{r}=\left(\Gamma_{r} \otimes I\right)(i d \otimes \tau)\left(\Gamma_{r} \otimes I\right), \quad \text { for all } r,
$$

then $\left(Q_{0} \otimes Q_{0}, \Theta\right)$ is a model for $(\mathcal{R}, H)$. Therefore there is a unital *-homomorphism $\Lambda_{0}: Q_{0} \longrightarrow Q_{0} \otimes Q_{0}$ such that

$$
\left(i d \otimes \Lambda_{0}\right)\left(\Gamma_{r}\right)=\Theta_{r}, \quad \text { for all } r .
$$

Hence each $\Gamma_{r}$ is a unitary corepresentation of $\left(Q_{0}, \Lambda_{0}\right)$.

We define a linear functional $h_{0}$ on $Q_{0}$ by

$$
h_{0}\left(\left[f_{r}\right]\right)= \begin{cases}1, & \text { if } f_{r}=\varepsilon^{\iota}, \\ 0, & \text { if } f_{r} \in B(H(r))^{*} \text { with } r \neq \iota .\end{cases}
$$

Proposition 3.2. Let $r, s \in \mathcal{J}(\mathcal{R})$ and $r \neq s$.

(i) For any $f_{r} \in B(H(r))^{*}$ and $f_{s} \in B(H(s))^{*}$,

$$
h_{0}\left(\left[f_{r}\right]^{*}\left[f_{s}\right]\right)=0 .
$$


(ii) For any $f_{r}, g_{r} \in B(H(r))^{*}$,

$$
h_{0}\left(\left[g_{r}\right]^{*}\left[f_{r}\right]\right)=\operatorname{dim}(H(r))^{-1} \sum_{i, j} \overline{g_{r}\left(e_{i, j}^{r}\right)} f_{r}\left(e_{i, j}^{r}\right)
$$

(iii) For any nonzero $a=\sum_{r}\left[f_{r}\right] \in Q_{0}$,

$$
h_{0}\left(a^{*} a\right)=\sum_{r} \operatorname{dim}(H(r))^{-1} \sum_{i, j}\left|f_{r}\left(e_{i, j}^{r}\right)\right|^{2}>0 .
$$

(iv) For any $i, j, k, l=1, \ldots, \operatorname{dim}(H(r))$,

$$
h_{0}\left(\left[\varepsilon_{i, j}^{r}\right]^{*}\left[\varepsilon_{k, l}^{r}\right]\right)=\operatorname{dim}(H(r))^{-1} \delta_{i, k} \delta_{j, l} .
$$

Proof: (i) We write

$$
\Gamma_{\tilde{r} s}=\sum_{k}\left(H\left(\mu_{i}\right) \otimes I\right) \Gamma_{r_{i}}\left(H\left(\mu_{i}^{*}\right) \otimes I\right),
$$

where $\mu_{i} \in \operatorname{Hom}\left(r_{i}, \tilde{r} s\right)$ such that $\sum_{i} \mu_{i} \mu_{i}^{*}=I_{\tilde{r} s}$, and $r_{i} \in \mathcal{J}(\mathcal{R})$. We have

$$
\begin{aligned}
h_{0}\left(\left[f_{r}\right]^{*}\left[f_{s}\right]\right) & =h_{0}\left(\left(\tilde{f}_{r} \otimes f_{s} \otimes i d\right)\left(\Gamma_{\tilde{r} s}\right)\right) \\
& =\left(\tilde{f}_{r} \otimes f_{s}\right)\left(\sum_{r_{i}=\iota} H\left(\mu_{i}\right) H\left(\mu_{i}^{*}\right)\right) .
\end{aligned}
$$

Note that for any objects $r, s$ and $t, \mu \longmapsto\left(I_{\tilde{r}} \diamond \mu\right)\left(\vartheta_{\tilde{r}} \diamond I_{t}\right)$ is a bijective linear map from $\operatorname{Hom}(r t, s)$ onto $\operatorname{Hom}(t, \tilde{r} s)$. Since $\operatorname{Hom}(r, s)=\{0\}$, it follows that $H\left(\mu_{i}\right)=0$ for all $i$ with $r_{i}=\iota$. This proves (i).

(ii) By arguments similar to those in (i) with $r=s$, we get

$$
h_{0}\left(\left[g_{r}\right]^{*}\left[f_{r}\right]\right)=\left(\widetilde{g}_{r} \otimes f_{r}\right)\left(\sum_{r_{i}=\iota} H\left(\mu_{i}\right) H\left(\mu_{i}^{*}\right)\right) .
$$

Since $\operatorname{Hom}(\iota, \tilde{r} r)=\mathbf{C} \bar{\vartheta}_{r}^{*}$ and $\operatorname{Hom}(\widetilde{r} r, \iota)=\mathbf{C} \bar{\vartheta}_{r}$, it follows that

$$
\sum_{r_{i}=\iota} H\left(\mu_{i}\right) H\left(\mu_{i}^{*}\right)=c H\left(\bar{\vartheta}_{r}^{*}\right) H\left(\bar{\vartheta}_{r}\right)
$$

for some scalar $c$. For any $i$ with $r_{i} \neq \iota$, we have $\mu_{i}^{*} \bar{\vartheta}_{r}^{*} \in \operatorname{Hom}\left(\iota, r_{i}\right)=\{0\}$. Hence

$$
\begin{aligned}
\bar{t}_{r}^{*} & =H\left(\bar{\vartheta}_{r}^{*}\right)=H\left(\sum_{i} \mu_{i} \mu_{i}^{*} \bar{\vartheta}_{r}^{*}\right)=H\left(\sum_{r_{i}=\iota} \mu_{i} \mu_{i}^{*} \bar{\vartheta}_{r}^{*}\right) \\
& =c H\left(\bar{\vartheta}_{r}^{*}\right) H\left(\bar{\vartheta}_{r}\right) H\left(\bar{\vartheta}_{r}^{*}\right)=c \bar{t}_{r}^{*} \bar{t}_{r} \bar{t}_{r}^{*} .
\end{aligned}
$$


Thus $c \bar{t}_{r} \bar{t}_{r}^{*}(1)=1$. Note that

$$
\begin{aligned}
\bar{t}_{r} \bar{t}_{r}^{*}(1) & =\sum_{i}\left\langle J_{r}^{-1}\left(e_{i}^{\tilde{r}}\right) \mid J_{r}^{-1}\left(e_{i}^{\tilde{r}}\right)\right\rangle=\operatorname{dim}(H(r)), \\
\bar{t}_{r}^{*} \bar{t}_{r} & =\sum_{i, j} J_{r} e_{i, j}^{r} J_{r}^{-1} \otimes e_{i, j}^{r}=\sum_{i, j} \widetilde{e_{i, j}^{r}} \otimes e_{i, j}^{r} .
\end{aligned}
$$

Now (ii) follows from (1), (2) and (3).

(iii) It is a consequence of (i) and (ii).

(iv) Using (ii), we get

$$
\begin{aligned}
h_{0}\left(\left[\varepsilon_{i, j}^{r}\right]^{*}\left[\varepsilon_{k, l}^{r}\right]\right) & =\operatorname{dim}(H(r))^{-1} \sum_{p, q} \overline{\varepsilon_{i, j}^{r}\left(e_{p, q}^{r}\right)} \varepsilon_{k, l}^{r}\left(e_{p, q}^{r}\right) \\
& =\operatorname{dim}(H(r))^{-1} \varepsilon_{k, l}^{r}\left(e_{i, j}^{r}\right) .
\end{aligned}
$$

For each $a \in Q_{0}$, we put

$\|a\|=\sup \{\|\pi(a)\|: \pi$ is a nondegenerate representation on Hilbert spaces $\}$.

Then $\|a\|$ is finite, and then $\|\cdot\|$ is a $C^{*}$-seminorm on $Q_{0}$. The ideal $\mathcal{I}_{0}$ of $Q_{0}$ consisting of elements of seminorm zero is closed under the involution operation *. The canonical quotient map $q: Q_{0} \longrightarrow Q_{0} / \mathcal{I}_{0}$ is a unital *-homomorphism. Let $Q$ denote the completion of $Q_{0} / \mathcal{I}_{0}$. We represent the $C^{*}$-algebra $Q$ on a Hilbert space by a faithful nondegenerate representation, and then see that

$$
\left\|(q \otimes q) \circ \Lambda_{0}(a)\right\| \leqslant\|a\|, \quad \forall a \in Q_{0} .
$$

We then deduce that there is a comultiplication $\Lambda: Q \longrightarrow Q \otimes Q$ such that

$$
\Lambda(q(a))=(q \otimes q) \circ \Lambda_{0}(a), \quad \forall a \in Q_{0} .
$$

Each $\beta_{r}=(i d \otimes q)\left(\Gamma_{r}\right)$ is a unitary corepresentation of $(Q, \Lambda)$, and the matrix elements of the family $\left\{\beta_{r}\right\}$ generate $Q$. By Remark 2.6(b) there exists a unique Haar measure $h$ for $\left(Q, \Lambda,\left\{\beta_{r}\right\}\right)$. Let $r \in \mathcal{J}(\mathcal{R})$ with $r \neq \iota$. Since the unit $I$ of $Q_{0}$ is not in $\mathcal{I}_{0}$, it follows that $q(I)$ is not in the linear span of $q\left(\left[\varpi_{i, j}^{r}\right]\right)$ for all $i, j$. By the Hahn-Banach theorem, we can choose $\eta \in Q^{*}$ such that $\eta(q(I))=1$ and $\eta\left(q\left(\left[\varpi_{i, j}^{r}\right]\right)\right)=0$ for all $i, j$. Using Theorem 2.3, we get

$$
\begin{aligned}
h\left(q\left(\left[\varpi_{i, j}^{r}\right]\right)\right) & =(\eta * h)\left(q\left(\left[\varpi_{i, j}^{r}\right]\right)\right) \\
& =\sum_{k} \eta\left(q\left(\left[\varpi_{i, k}^{r}\right]\right)\right) h\left(q\left(\left[\varpi_{k, j}^{r}\right]\right)\right)=0 .
\end{aligned}
$$


Therefore $h \circ q=h_{0}$. If $\pi$ is the cyclic representation of $Q$ induced by the positive linear functional $h$, then it follows from Proposition 3.2(iii) that $\pi \circ q$ is a faithful nondegenerate representation of $Q_{0}$. Therefore $\mathcal{I}_{0}=\{0\}$ and $\|\cdot\|$ is a $C^{*}$-norm.

We define an antimultiplicative linear map $\jmath: Q_{0} \longrightarrow Q_{0}$ by

$$
\jmath\left(\left[f_{r}\right]\right)=\left(f_{r} \otimes i d\right)\left(\Gamma_{r}^{*}\right) .
$$

Then $\jmath(\jmath(a))=a$ and $\jmath\left(a^{*}\right)=\jmath(a)^{*}$ for all $a \in A_{0}$, and $(i d \otimes \jmath)\left(\Gamma_{r}\right)=\Gamma_{r}^{*}$ for all $r$.

We summarise the above discussion in the following result.

THEOREM 3.3. With the above notation, $Q=(Q, \Lambda, \mathcal{L}, \jmath)$ is a compact quantum group, where $\mathcal{L}=\left\{\Gamma_{r}: r \in \mathcal{J}(\mathcal{R})\right\}$.

We define a functor $\widehat{H}: \mathcal{R} \longrightarrow \mathcal{U}_{\text {corep }_{f}}(Q)$ by

$$
\widehat{H}(r)=\Gamma_{r} \quad \text { and } \quad \hat{H}(\mu)=H(\mu) .
$$

THEOREM 3.4. With the above notation:

(i) $\widehat{H}$ is a fully faithful monoidal linear *-functor.

(ii) The categories $\mathcal{R}$ and $\mathcal{U}$ corep $_{f}(Q)$ are equivalent.

Proof: (i) It is easy to show that $\widehat{H}$ is a faithful monoidal linear *-functor. To prove that $\widehat{H}$ is full, let $T \in B(H(r), H(s))$ such that

$$
(T \otimes I) \Gamma_{r}=\Gamma_{s}(T \otimes I) .
$$

We write

$$
\begin{aligned}
& \Gamma_{r}=\sum_{k}\left(H\left(\mu_{k}\right) \otimes I\right) \Gamma_{r_{k}}\left(H\left(\mu_{k}^{*}\right) \otimes I\right), \\
& \Gamma_{s}=\sum_{l}\left(H\left(\nu_{l}\right) \otimes I\right) \Gamma_{s_{l}}\left(H\left(\nu_{l}^{*}\right) \otimes I\right),
\end{aligned}
$$

where $\mu_{k} \in \operatorname{Hom}\left(r_{k}, r\right)$ with $\sum_{k} \mu_{k} \mu_{k}^{*}=I_{r}$, and $\nu_{l} \in \operatorname{Hom}\left(s_{l}, s\right)$ with $\sum_{l} \nu_{l} \nu_{l}^{*}=I_{s}$, and $r_{k}, s_{l} \in \mathcal{J}(\mathcal{R})$. We have

$$
\left(H\left(\nu_{l}^{*}\right) T H\left(\mu_{k}\right) \otimes I\right) \Gamma_{r_{k}}=\Gamma_{s_{l}}\left(H\left(\nu_{l}^{*}\right) T H\left(\mu_{k}\right) \otimes I\right) .
$$

It then follows that

$$
\sum_{i, j} H\left(\nu_{l}^{*}\right) T H\left(\mu_{k}\right) \theta_{i, j}^{r_{k}} \otimes\left[\varpi_{i, j}^{r_{k}}\right]=\sum_{m, n} \theta_{m, n}^{s_{l}} H\left(\nu_{l}^{*}\right) T H\left(\mu_{k}\right) \otimes\left[\varpi_{m, n}^{s_{l}}\right]
$$


If $s_{l} \neq r_{k}$, choose a linear functional $\eta$ on $Q_{0}$ such that

$$
\eta\left(\left[\varpi_{m, n}^{t}\right]\right)=\delta_{r_{k}, t} \delta_{m, n}, \quad \forall m, n=1, \ldots, \operatorname{dim}(H(t)),
$$

for all $t \in \mathcal{J}(\mathcal{R})$. We then deduce that $H\left(\nu_{l}^{*}\right) T H\left(\mu_{k}\right)=0$. If $s_{l}=r_{k}$ then

$$
H\left(\nu_{l}^{*}\right) T H\left(\mu_{k}\right) \theta_{i, j}^{r_{k}}=\theta_{i, j}^{r_{k}} H\left(\nu_{l}^{*}\right) T H\left(\mu_{k}\right),
$$

for all $i, j=1, \ldots, \operatorname{dim}\left(H\left(r_{k}\right)\right)$. It then follows that $H\left(\nu_{l}^{*}\right) T H\left(\mu_{k}\right)$ commutes with all elements of $B\left(H\left(r_{k}\right)\right)$, and hence $H\left(\nu_{l}^{*}\right) T H\left(\mu_{k}\right)=c(k, l) I_{H\left(r_{k}\right)}$ for some scalar $c(k, l)$. Now we get

$$
T=\widehat{H}\left(\sum_{s_{l}=r_{k}} c(k, l) \nu_{l} \mu_{k}^{*}\right)
$$

and this proves that $\widehat{H}$ is full.

(ii) The family $\mathcal{L}$ consists of mutually nonequivalent irreducible corepresentations of $Q$, and the matrix elements of all elements of $\mathcal{L}$ generate $Q_{0}$. By Theorem 2.4, every object $\alpha$ of $\mathcal{U}$ corep $_{f}(Q)$ is the direct sum of irreducible unitary subcorepresentations $\alpha_{i}$. By Theorem 2.5, each $\alpha_{i}$ is equivalent to an element $\Gamma_{r_{i}}$ of $\mathcal{L}$. If we put $r=\sum_{i}^{\oplus} r_{i}$, then $\widehat{H}(r)=\Gamma_{r}$ is equivalent to $\sum_{i}^{\oplus} \Gamma_{r_{i}}$. Therefore $\alpha$ is equivalent to $\widehat{H}(r)$, and hence $\widehat{H}$ is an equivalence.

\section{The orthogonality Relations and the Plancherel theorem}

In this section $A=(A, \Delta, F, \kappa)$ is a compact quantum group, and $\lambda$ is the Haar measure for $A$. Let $\mathcal{J}$ be a complete set of mutually nonequivalent irreducible unitary corepresentations belonging to $\mathcal{R}_{F}$, where $\mathcal{R}_{F}$ was defined just before Proposition 1.1. We assume that the trivial one dimensional corepresentation $\iota=1 \otimes I$ is in $\mathcal{J}$. For each $\left(\alpha, V_{\alpha}\right)$ in $\mathcal{J}$, we pick an orthonormal basis $\left\{e_{i}^{\alpha}\right\}$ for $V_{\alpha}$. Let $J_{\alpha}: V_{\alpha} \rightarrow V_{\tilde{\alpha}}$ be the bijective antilinear map defining the conjugate $\widetilde{\alpha}=\alpha^{J_{\alpha} \otimes *}$ of $\alpha$. We define

$$
e_{i, j}^{\alpha}(u)=\left\langle u \mid e_{j}^{\alpha}\right\rangle J_{\alpha}^{-1}\left(e_{i}^{\tilde{\alpha}}\right), \quad \forall u \in V_{\alpha}
$$

Then $\left\{e_{i, j}^{\alpha}\right\}$ is a basis for $B\left(V_{\alpha}\right)$. We denote by $\left\{\varepsilon_{i, j}^{\alpha}\right\}$ the dual basis of $\left\{e_{i, j}^{\alpha}\right\}$ for $B(H(r))^{*}$. We write

$$
\alpha=\sum_{i, j} e_{i, j}^{\alpha} \otimes \alpha(i, j)
$$

Then the set

$$
\{\alpha(i, j): i, j=1, \ldots, \operatorname{dim}(\alpha), \alpha \in \mathcal{J}\}
$$


is a basis for the vector space $A_{F}$, where $A_{F}$ was defined in Proposition 1.1. We define

$$
\theta_{i, j}^{\alpha}(u)=\left\langle u \mid e_{j}^{\alpha}\right\rangle e_{i}^{\alpha}, \quad \forall u \in V_{\alpha} .
$$

We denote by $\left\{\varpi_{i, j}^{\alpha}\right\}$ the dual basis of $\left\{\theta_{i, j}^{\alpha}\right\}$ for $B(H(r))^{*}$.

The following result is the orthogonality relations for our compact quantum groups; see [5, Chapter IX]. It improves the orthogonality relations of [14, Theorem 5.7.4].

TheOREM 4.1. For any $\left(\alpha, V_{\alpha}\right)$ and $\left(\beta, V_{\beta}\right)$ in $\mathcal{J}$, we have

$$
\lambda\left(\alpha(i, j)^{*} \beta(k, l)\right)=\operatorname{dim}(\alpha)^{-1} \delta_{\alpha \beta} \delta_{i k} \delta_{j l},
$$

for all $i, j=1, \ldots, \operatorname{dim}(\alpha)$ and $k, l=1, \ldots, \operatorname{dim}(\beta)$.

ProOF: Let $\mathcal{R}=\mathcal{U} \operatorname{corep}_{f}\left(A_{F}\right)$. Then $\mathcal{R}$ is a left autonomous strict monoidal $C^{*}$-category with subobjects and direct sums. The forgetful functor $H\left(\alpha, V_{\alpha}\right)=V_{\alpha}$ is a faithful monoidal linear ${ }^{*}$-functor. Let $Q_{0}, \Gamma$ and $\Lambda$ be constructed as in Theorem 3.3. Observe that $\mathcal{J}$ is a complete set of mutually nonequivalent irreducible objects of the category $\mathcal{R}$. We define a model $(A, \Pi)$ for $(\mathcal{R}, H)$ by

$$
\Pi_{\alpha}=\alpha, \quad \text { for all } \alpha \in \mathcal{R} .
$$

Since $\left(Q_{0}, \Gamma\right)$ is a universal model, there is a homomorphism $\Phi: Q_{0} \longrightarrow A_{F}$ of unital *-algebras such that $\Phi\left(\left[\varepsilon_{i, j}^{\alpha}\right]\right)=\alpha(i, j)$ for all $i, j=1, \ldots, \operatorname{dim}\left(V_{\alpha}\right)$ and $\alpha$ in $\mathcal{J}$. Now we write

$$
\alpha=\sum_{i, j} \theta_{i, j}^{\alpha} \otimes \alpha_{\theta}(i, j)
$$

We then deduce that $\Phi\left(\left[\varpi_{i, j}^{\alpha}\right]\right)=\alpha_{\theta}(i, j)$ for all $i, j$. Let $\left(\alpha, V_{\alpha}\right)$ in $\mathcal{J}$ with $\alpha \neq \iota$. By the Hahn-Banach theorem, we can choose $\eta \in A^{*}$ such that $\eta(I)=1$ and $\eta\left(\alpha_{\theta}(i, j)\right)=$ 0 for all $i, j$. Using Theorem 2.3, we get

$$
\begin{aligned}
\lambda\left(\Phi\left(\left[\varpi_{i, j}^{\alpha}\right]\right)\right) & =(\eta * \lambda)\left(\alpha_{\theta}(i, j)\right) \\
& =\sum_{k} \eta\left(\alpha_{\theta}(i, k)\right) \lambda\left(\alpha_{\theta}(k, j)\right)=0,
\end{aligned}
$$

for all $i, j$. Therefore $\lambda \circ \Phi=h_{0}$ on $Q_{0}$, where $h_{0}$ was defined just before Proposition 3.2. It then follows that for any $\left(\alpha, V_{\alpha}\right)$ and $\left(\beta, V_{\beta}\right)$ in $\mathcal{J}$, we have

$$
\lambda\left(\alpha(i, j)^{*} \beta(k, l)\right)=h_{0}\left(\left[\varepsilon_{i, j}^{\alpha}\right]^{*}\left[\varepsilon_{k, l}^{\beta}\right]\right),
$$

for all $i, j=1, \ldots, \operatorname{dim}(\alpha)$ and $k, l=1, \ldots, \operatorname{dim}(\beta)$. Now using (i) and (iv) of Proposition 3.2 we get the desired formula. 
REMARK 4.2. The proof of Theorem 4.1 is dependent only on Theorem 3.3 and is independent of [14, Theorem 5.7 and Theorem 5.3]. To get the orthogonality relations of $[\mathbf{1 4}]$ we proceed as follows. If we write

$$
J_{\alpha}^{-1}\left(e_{i}^{\tilde{\alpha}}\right)=\sum_{k} c^{\alpha}(k, i) e_{k}^{\alpha},
$$

then $e_{i, j}^{\alpha}=\sum_{k} c^{\alpha}(k, i) \theta_{k, j}^{\alpha}$. It then follows that

$$
\sum_{i, j} e_{i, j}^{\alpha} \otimes \alpha(i, j)=\sum_{k, j} \theta_{k, j}^{\alpha} \otimes\left\{\sum_{i} c^{\alpha}(k, i) \alpha(i, j)\right\}
$$

Therefore the matrix elements of $\alpha$ with respect to the orthonormal basis $\left\{e_{i}^{\alpha}\right\}$ are

$$
\alpha_{\theta}(i, j)=\sum_{p} c^{\alpha}(i, p) \alpha(p, j)
$$

We then deduce that

$$
\lambda\left(\alpha_{\theta}(i, j)^{*} \beta_{\theta}(k, l)\right)=\operatorname{dim}(\alpha)^{-1} \delta_{\alpha \beta} \delta_{j l} \sum_{p} \overline{c^{\alpha}(i, p)} c^{\beta}(k, p),
$$

which is essentially the formula of [14, Theorem 5.7.4]. In the same fashion, we can establish the orthogonality ralations for the matrix elements of $\left(\alpha, V_{\alpha}\right)$ in $\mathcal{J}$ with respect to any basis of $B\left(V_{\alpha}\right)$.

Now we define an inner product on $A_{F}$ by

$$
\langle a \mid b\rangle=\lambda\left(b^{*} a\right), \quad \forall a, b \in A_{F} .
$$

The completion of $A_{F}$ under the norm induced by this inner product is denoted by $L^{2}(\lambda)$. Let $\left(\alpha, V_{\alpha}\right)$ be an element of $\mathcal{J}$. The Hilbert subspace of $L^{2}(\lambda)$ generated by the matrix elements of $\alpha$ is denoted by $L_{\alpha}^{2}(\lambda)$. By the orthogonality relations, the set

$$
\left\{\operatorname{dim}(\alpha)^{1 / 2} \alpha(i, j): i, j=1, \ldots, \operatorname{dim}(\alpha)\right\}
$$

is an orthonormal basis for $L_{\alpha}^{2}(\lambda)$. Define an inner product on $X_{\alpha}=B\left(V_{\alpha}\right)$ so that the set

$$
\left\{\operatorname{dim}(\alpha)^{-1 / 2} e_{i, j}^{\alpha}: i, j=1, \ldots, \operatorname{dim}(\alpha)\right\}
$$

is an orthonormal basis for $X_{\alpha}$.

We define the Fourier transformation $\mathcal{F}_{\alpha}: L_{\alpha}^{2}(\lambda) \longrightarrow B\left(V_{\alpha}\right)$ by

$$
\mathcal{F}_{\alpha}\left(\xi_{\alpha}\right)=(i d \otimes \lambda)\left(\alpha^{i d \otimes *}\left(I \otimes \xi_{\alpha}\right)\right), \quad \forall \xi_{\alpha} \in L_{\alpha}^{2}(\lambda)
$$


where $\alpha^{i d \otimes *}$ denotes the image of $\alpha$ under the map $i d \otimes *$. Let $X$ denote the Hilbert space direct sum of all Hilbert spaces $X_{\alpha}$ with $\alpha \in \mathcal{J}$. Observe that $L^{2}(\lambda)$ is the Hilbert space direct sum of all Hilbert spaces $L_{\alpha}^{2}(\lambda)$ with $\alpha \in \mathcal{J}$. We define the Fourier transformation $\mathcal{F}: L^{2}(\lambda) \longrightarrow X$ by $\mathcal{F}=\sum_{\alpha \in \mathcal{J}}^{\oplus} \mathcal{F}_{\alpha}$.

The following result is an analogue of the Plancherel theorem for compact groups.

Proposition 4.3. The Fourier transformation $\mathcal{F}: L^{2}(\lambda) \longrightarrow X$ is a unitary. Proof: Observe that for each $\alpha$ in $\mathcal{J}$, we have

$$
\mathcal{F}_{\alpha}\left(\xi_{\alpha}\right)=\sum_{i, j}\left\langle\xi_{\alpha} \mid \alpha(i, j)\right\rangle e_{i, j}^{\alpha}, \quad \forall \xi_{\alpha} \in L_{\alpha}^{2}(\lambda)
$$

We compute

$$
\begin{aligned}
\left\|\mathcal{F}_{\alpha}\left(\xi_{\alpha}\right)\right\|^{2} & =\sum_{i, j, k, l}\left\langle\xi_{\alpha} \mid \alpha(i, j)\right\rangle \overline{\left\langle\xi_{\alpha} \mid \alpha(k, l)\right\rangle} \operatorname{dim}(\alpha) \delta_{i, k} \delta_{j, l} \\
& =\operatorname{dim}(\alpha) \sum_{i, j}\left|\left\langle\xi_{\alpha} \mid \alpha(i, j)\right\rangle\right|^{2} \\
\left\|\xi_{\alpha}\right\|^{2} & =\sum_{i, j}\left|\left\langle\xi_{\alpha} \mid \operatorname{dim}(\alpha)^{1 / 2} \alpha(i, j)\right\rangle\right|^{2} \\
& =\operatorname{dim}(\alpha) \sum_{i, j}\left|\left\langle\xi_{\alpha} \mid \alpha(i, j)\right\rangle\right|^{2}
\end{aligned}
$$

\section{REFERENCES}

[1] H.H. Bui, 'Morita equivalence of twisted crossed products by coactions', J. Funct. Anal. 123 (1994), 59-98.

[2] H.H. Bui, Compact quantum groups associated with monoidal functors, Proc. Amer. Math. Soc. (to appear).

[3] S. Baaj and G. Skandalis, 'Unitaires multiplicatifs et dualite pour les produits crossés de $C^{*}$-algèbres', Ann. Sci. École. Norm. Sup. 26 (1993), 425-488.

[4] S. Doplicher and J.E. Roberts, 'A new duality theory for compact groups', Invent. Math. 98 (1989), 157-218.

[5] J.M.G. Fell and R.S. Doran, Representations of *algebras, locally compact groups and Banach *-algebraic bundles, Vol. 2, Pure and Applied Math. 126 (Academic Press, New York, London, 1988).

[6] A. Joyal and R. Street, 'An introduction to the Tannaka duality and quantum groups', in Lecture Notes in Mathematics 1488 (Springer-Verlag, Berlin, Heidelberg, New York, 1991), pp. 411-492.

[7] A. Joyal and R. Street, 'Braided tensor categories', Adv. Math. 102 (1993), 20-78. 
[8] C. Kassel, Quantum groups, Graduate Texts in Mathematics 155 (Springer-Verlag, Berlin, Heidelberg, New York, 1995).

[9] C. Kassel and V. Turaev, 'Double construction for monoidal categories', Acta. Math. 175 (1995), 1-48.

[10] W. Rudin, Functional analysis (McGraw-Hill Book Co., New York, 1991).

[11] G. Skandalis, 'Operator algebras and duality', in Proceedings of International Congress of Mathematicians 2, 1990, pp. 997-1009.

[12] A. Van Daele, 'Dual pairs of Hopf *-algebras', Bull. London Math. Soc. 25 (1993), 209-230.

[13] A. Van Daele, 'The Haar measure in a compact quantum group', Proc. Amer. Math. Soc. 123 (1995), 3125-3128.

[14] S.L. Woronowicz, 'Compact matrix pseudogroups', Comm. Math. Phys. 111 (1987), 613-665.

[15] S.L. Woronowicz, 'Tannaka-Krein duality for compact matrix pseudogroups. Twisted $\mathrm{SU}(\mathrm{N})$ groups', Invent. Math. 93 (1988), 35-76.

[16] D.N. Yetter, 'Quantum groups and representation of monoidal categories', Math. Proc. Cambridge Philos. Soc. 180 (1990), 261-290.

School of Mathematics

The University of New South Wales

Sydney NSW 2052

Australia 